\title{
Atuação dos profissionais do consultório na rua no âmbito da Atenção Primária à Saúde do Brasil: Uma revisão de escopo
}

\author{
Practice of street clinic professionals in the context of Primary Health Care in Brazil: A scoping \\ review
}

Practica de los profesionales de consultorio en la calle en el ámbito de la Atención Primaria de Salud de Brasil: Una revisión de alcance

\section{Resumo}

Objetivo: mapear na literatura científica nacional as produções relacionadas à atuação dos profissionais do Consultório na Rua (CnaR) no âmbito da Atenção Primária à Saúde (APS) no Brasil. Método: Trata-se de uma revisão de escopo que seguiu as recomendações propostas pelo Manual de Evidências do Instituto Joanna Briggs (JBI). A aplicação da estratégia População, Conceito, Contexto (PCC) culminou na seguinte questão de investigação: "O que se sabe sobre a atuação dos profissionais do CnaR no âmbito da APS no Brasil?’. A busca ocorreu entre dezembro de 2020 e janeiro de 2021, utilizando-se o descritor "Consultório na rua". Após aplicar os critérios de elegibilidade, foram selecionados treze estudos. Na extração dos dados empregou-se o instrumento proposto pelo JBI. Resultados: A amostra final foi composta de treze estudos. Para melhor compreensão, os estudos mapeados foram organizados em eixos, a saber: assistência à saúde; articulação com os dispositivos das Redes de Atenção à Saúde e Redes Intersetoriais; tecnologias leves de cuidado; redução de danos; e trabalho em equipe. Conclusão: O total de publicações incluídas nesta revisão de escopo demonstra que a temática em estudo tem sido pouco discutida. Nesse contexto, diante dos achados suscita-se a demanda por pesquisas que tenham como finalidade elucidar a atuação dos profissionais do CnaR. Os resultados do estudo promovem inovação, divulgação e disseminação dessa temática que é de grande relevância para a saúde pública no Brasil, uma vez que elenca e mapeia a atuação dos referidos profissionais.

Palavras-chave: Atenção primária à saúde; Pessoas em situação de rua; Consultório na rua; Prática profissional. 


\begin{abstract}
Objective: to map in the national scientific literature the productions related to the performance of the Consultório na Rua (CnaR) professionals in the scope of Primary Health Care (PHC) in Brazil. Method: This is a scope review that followed the recommendations proposed by the Joanna Briggs Institute's Evidence Manual (JBI). The application of the Population, Concept, Context (PCC) strategy culminated in the following research question: "What is known about the performance of CnaR professionals in the context of PHC in Brazil?". The search took place between December 2020 and January 2021, using the descriptor "Consultório na rua". After applying the eligibility criteria, thirteen studies were selected. In the extraction of data, the instrument proposed by JBI was used. Results: The sample consisted of thirteen studies. For better understanding, the mapped studies were organized into axes, namely: health care; articulation with the devices of the Health Care Networks and Intersectoral Networks; lightweight care technologies; harm reduction; and teamwork. Conclusion: The total number of publications included in this scope review shows that the topic under study has been little discussed. In this context, given the findings, there is a demand for research aimed at elucidating the performance of CnaR professionals. The results of the study promote innovation, dissemination and dissemination of this theme that is of great relevance to public health in Brazil, since it lists and maps the performance of these professionals.
\end{abstract}

Keywords: Primary health care; Homeless people; Street office; Professional practice.

\title{
Resumen
}

Objetivo: mapear en la literatura científica nacional las producciones relacionadas con el desempeño de los profesionales del Consultório na Rua (CnaR) en el ámbito de la Atención Primaria de Salud (APS) en Brasil. Método: Se trata de una revisión del alcance que siguió las recomendaciones propuestas por el Manual de Evidencia (JBI) del Instituto Joanna Briggs. La aplicación de la estrategia Población, Concepto, Contexto (PCC) culminó en la siguiente pregunta de investigación: "¿Qué se sabe sobre el desempeño de los profesionales de la CnaR en el contexto de la APS en Brasil?". La búsqueda se realizó entre diciembre de 2020 y enero de 2021, utilizando el descriptor "Consultório na rua". Después de aplicar los criterios de elegibilidad, se seleccionaron trece estudios. En la extracción de datos se utilizó el instrumento propuesto por JBI. Resultados: La muestra consistió em trece estúdios. Para una mejor comprensión, los estudios mapeados se organizaron en ejes, a saber: atención de la salud; articulación con los dispositivos de las Redes de Atención en Salud y Redes Intersectoriales; tecnologías de cuidado ligero; reducción de daños; y trabajo en equipo. Conclusión: El número total de publicaciones incluidas en esta revisión del alcance muestra que el tema en estudio ha sido poco discutido. En este contexto, ante los hallazgos, existe una demanda de investigación orientada a dilucidar el desempeño de los profesionales de la CnaR. Los resultados del estudio promueven la innovación, difusión y difusión de este tema de gran relevancia para la salud pública en Brasil, ya que enumera y mapea el desempeño de estos profesionales.

Palabras clave: Atención primaria de salud; Gente sin hogar; Consultorio en la calle; Practica professional.

\section{Introdução}

A População em Situação de Rua (PSR) é um grupo heterogêneo que tem como características: a ausência de domicílios convencionais regulares e fixos, vínculos familiares totalmente fragilizados ou rompidos (Brasil, 2014), a pobreza, as precárias condições de vida, a dificuldade de acesso aos serviços básicos de saúde e outros direitos de cidadania (Engstrom et al., 2019). Trata-se de um grupo social que vivencia múltiplas vulnerabilidades marcadas por processos de exclusão social, marginalização e preconceitos (Teixeira et al., 2019).

Valle e Sarah (2020) afirmam que os passos estabelecidos pelas políticas públicas para a PSR ainda são tímidos, entretanto, sinalizam a importância da construção da Política Nacional para População em Situação de Rua (PNPSR), em 2009, como um avanço para superação da invisibilidade social que acompanha este grupo. A referida política permitiu que se desvelasse o processo de invisibilização histórica nesse contexto, prevendo ações interministeriais para incentivar estratégias públicas em todas as esferas de governo (Vale \& Vecchia, 2019). No que se refere à atenção à saúde, o desdobramento da PNPSR culminou em 2011 na instituição do Consultório na Rua (CnaR) enquanto equipe de Atenção Básica (eAB) para população específica, por meio da publicação da Política Nacional da Atenção Básica (PNAB) (Brasil, 2011; Brasil, 2017).

As equipes de Consultório na Rua (eCR) estão inseridas em um campo de atuação bastante complexo e permeado por múltiplas vulnerabilidades. Esse dispositivo de produção de saúde foi criado para melhorar a capacidade de resposta às demandas e necessidades inerentes a PSR (Hallais \& Barros, 2015). Cabe a essas equipes prestar atenção à saúde em um cenário 
diferenciado e dinâmico e devem ser capazes de articular a AB, a Atenção Psicossocial e mediações socioculturais (Cardoso et al., 2018; Santos \& Ceccim, 2018). Nesse ínterim, as eCR são fundamentais para a mitigação das iniquidades em saúde e, consequentemente, consolidam na prática o direito constitucional de acesso à saúde bem como os princípios doutrinários do Sistema Único de Saúde (SUS), a Universalidade, a Equidade e a Integralidade.

A complexidade da atuação das eCR junto à PSR sugere a adoção de modelos centrados em uma visão ampliada de cuidado considerando singularidades, necessidades e contextos de vida (Engstrom et al., 2019). Diante da conjuntura apresentada, foi observado a importância de elucidar as características da atuação com vistas ao desenvolvimento de um melhor entendimento sobre a práxis profissional que marca o trabalho em saúde a PSR. Cardoso et al. (2019) observaram ainda que há uma escassez de estudos relativos ao processo de trabalho dos profissionais do CnaR. Similarmente, Vale e Vecchia (2019) também mencionam a respeito da produção acadêmica incipiente justificada em parte por se tratar de uma política relativamente recente.

Destaca-se que não foram encontradas na literatura científica revisões sistemáticas sobre a atuação dos profissionais do CnaR no âmbito da APS do Brasil. Este fato revela a necessidade de identificar as principais características relacionadas ao conceito dessa atuação. Ademais, justifica-se a realização dessa pesquisa pela necessidade de preencher os gaps da literatura. Diante do exposto e considerando as complexidades da atuação das eCR junto à PSR, o presente estudo tem como objetivo mapear na literatura científica nacional as produções relacionadas à atuação dos profissionais do CnaR no âmbito da APS do Brasil.

\section{Metodologia}

Trata-se de uma revisão de escopo que seguiu as recomendações propostas pelo Manual de Evidências do Instituto Joanna Briggs (JBI, 2020). O desenvolvimento desta revisão percorreu seis passos metodológicos: (1) identificação da questão de pesquisa, (2) identificação de estudos relevantes, (3) seleção dos estudos, (4) extração de dados, (5) separação, sumarização e relatório de resultados e (6) divulgação dos resultados (Barbiani, Nora \& Schaefer, 2016). Para elaboração da questão de investigação foi utilizada a estratégia População, Conceito, Contexto (PCC) na qual, os profissionais do Consultório na Rua constituíram a população, a atuação destes o conceito e o âmbito da APS no Brasil como o contexto. A aplicação dessa estratégia culminou na seguinte questão de investigação: O que se sabe sobre a atuação dos profissionais do CnaR no âmbito da APS no Brasil?

O primeiro passo dado teve o intuito de rastrear e identificar outras revisões ou protocolos de revisão semelhantes ao objetivo deste estudo. Para tanto, foi realizada em dezembro de 2020 busca na biblioteca Cochrane e no banco de dados International Prospective Register of Systematic (PROSPERO), todavia, não foram identificados registros que abordassem a temática proposta.

Com intuito de selecionar a estratégia de busca, em princípio foi feita uma busca não controlada na Biblioteca Virtual em Saúde (BVS) e no Google Acadêmico observando as palavras do título, resumo e termos indexados, para a delimitação de descritores por meio do Descritores de Ciências da Saúde (DeCS), optando-se pela palavra-chave "Consultório na rua". Os critérios de inclusão da amostra foram: textos completos disponibilizados na íntegra e publicados em fontes indexadas, nos idiomas português, inglês e espanhol. Como critérios de exclusão: textos repetidos nas bases de dados e os que não respondiam à questão de investigação.

Para selecionar a amostra foram seguidas algumas etapas: primeira, busca controlada na BVS e a leitura dos títulos e resumos; segunda, a leitura dos estudos na íntegra; e, posteriormente a busca nas referências dos estudos selecionados por outros que pudessem ser acrescentados na amostra. O instrumento de extração de dados foi adaptado do JBI, contendo os itens: autoria, 
ano, periódico, estado, objetivo, tipo de estudo/abordagem, população/participantes e principais resultados da atuação. A apresentação dos resultados se deu mediante categorização.

Como se trata de uma revisão de escopo em que compuseram a amostra artigos científicos já publicados, afirma-se que não houve necessidade de avaliação por um comitê de ética em pesquisa. Para seleção da amostra foi realizada a leitura criteriosa de títulos, resumos e palavras-chave. Nas ocasiões em que a leitura inicial era insuficiente para definir a seleção, realizou-se a leitura do artigo na íntegra. Os artigos foram inseridos no gerenciador de referências Mendeley para a identificação de duplicatas. Os elementos desta revisão foram verificados a partir do Checklist e o fluxograma Preferred Reporting Items for Systematic reviews and Meta-Analyses extension for Scoping Reviews (PRISMA-ScR).

\section{Resultados}

O fluxograma do processo de seleção e inclusão dos estudos, baseado no PRISMA-ScR, encontra-se na Figura 1. A pesquisa na BVS resultou em 101 registros. Destes, 14 foram excluídos por não estarem disponíveis na íntegra. Após a leitura dos títulos e resumos dos estudos encontrados, observou-se que 65 não atenderam aos critérios de elegibilidade. Dos 22 eleitos para leitura na íntegra, 10 não responderam à questão de investigação. As referências dos estudos selecionados para a amostra foram verificadas com a finalidade de encontrar artigos que pudessem ser acrescentados. Entretanto, apenas um que respondeu à questão de investigação não se encontrava incluído na amostra final, os demais foram excluídos.

Figura 1. Fluxograma do processo de seleção e inclusão dos estudos baseado no PRISMA-ScR (adaptado). Maceió, Alagoas, 2021.

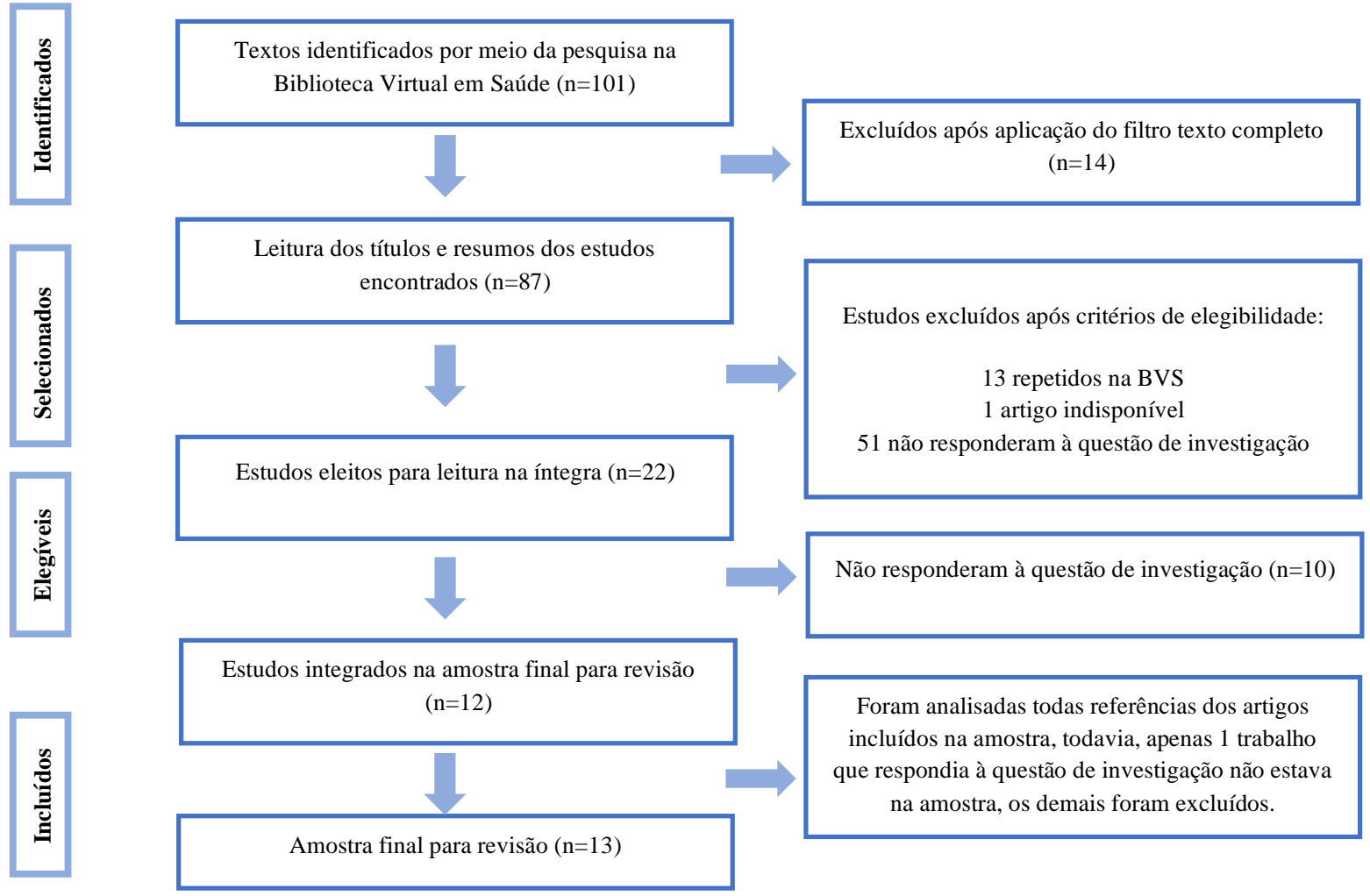

Fonte: Dados da pesquisa (2021).

O Quadro 1 apresenta a caracterização dos estudos selecionados quanto a autoria, ano, periódico, estado, objetivos, tipo de estudo/abordagem e população/participantes. $\mathrm{O}$ total de estudos que compuseram a amostra $(\mathrm{n}=13)$ foram resgatados nas 
bases de dados LILACS ( $n=7), \operatorname{BDENF}(n=5)$ e MEDLINE ( $n=1)$ via BVS. Estes foram publicados entre os anos 2015 e 2020 , observando-se maior número de publicações nos anos de $2018(n=4)$ e $2019(n=3)$. A maioria dos estudos foram realizados nas Regiões Sudeste (n=6) e Centro-Oeste (n=3) o que representaram juntas 69,29\% da amostra; seguida do Nordeste (n=2) $15,38 \%$ e as Regiões Norte $(n=1)$ e Sul $(n=1)$ com apenas 7,69\% cada. Todos utilizaram a abordagem qualitativa de pesquisa, destacandose as do tipo exploratório $(n=5)$ e relatos de experiência $(n=3)$.

Quadro 1. Caracterização dos estudos selecionados quanto a autoria, ano, periódico, estado, objetivos, tipo de estudo/abordagem e população/participantes. Maceió, Alagoas, 2021.

\begin{tabular}{|c|c|c|c|c|c|c|c|}
\hline Estudo & Autoria & Ano & Periódico & Estado & Objetivos & $\begin{array}{c}\text { Tipo de } \\
\text { estudo/Abordage } \\
\text { m }\end{array}$ & $\begin{array}{c}\text { População/participa } \\
\text { ntes }\end{array}$ \\
\hline E1 & Souza et al. & 2020 & $\begin{array}{l}\text { Revista do } \\
\text { Nufen }\end{array}$ & Goiás & $\begin{array}{l}\text { Relatar a experiência de } \\
\text { ações } \\
\text { desenvolvidas pelo CnaR } \\
\text { para a redução de } \\
\text { vulnerabilidade } \\
\text { efetividade do cuidado }\end{array}$ & $\begin{array}{c}\text { Relato de } \\
\text { Experiência/Quali } \\
\text { tativa }\end{array}$ & $\begin{array}{c}\text { Duas eCR de } \\
\text { Aparecida de Goiânia }\end{array}$ \\
\hline E2 & $\begin{array}{l}\text { Timóteo et } \\
\text { al. }\end{array}$ & 2020 & $\begin{array}{l}\text { Enfermage } \\
\mathrm{m} \text { em Foco }\end{array}$ & Alagoas & $\begin{array}{l}\text { Caracterizar o trabalho e as } \\
\text { ações desenvolvidas pelas } \\
\text { eCR de Maceió }\end{array}$ & $\begin{array}{l}\text { Estudo } \\
\text { exploratório/Quali } \\
\text { tativa }\end{array}$ & $\begin{array}{l}\text { Treze profissionais de } \\
\text { ensino superior, } \\
\text { médio e técnico que } \\
\text { atuam no CnaR de } \\
\text { Maceió }\end{array}$ \\
\hline E3 & $\begin{array}{l}\text { Engstrom et } \\
\text { al. }\end{array}$ & 2019 & $\begin{array}{l}\text { Saúde } \\
\text { Debate }\end{array}$ & $\begin{array}{l}\text { Rio de } \\
\text { Janeiro }\end{array}$ & $\begin{array}{l}\text { Analisar a produção de } \\
\text { cuidados primários à saúde à } \\
\text { população em situação de } \\
\text { rua, prestados por eCR no } \\
\text { contexto de uma metrópole } \\
\text { brasileira, identificando } \\
\text { potencialidades } \\
\text { dificuldades }\end{array}$ & $\begin{array}{c}\text { Estudo de } \\
\text { caso/Qualitativa }\end{array}$ & $\begin{array}{l}\text { Sete eCR do } \\
\text { município do Rio de } \\
\text { Janeiro }\end{array}$ \\
\hline E4 & $\begin{array}{c}\text { Vale e } \\
\text { Vecchia }\end{array}$ & 2019 & $\begin{array}{l}\text { Estudos de } \\
\text { Psicologia }\end{array}$ & $\begin{array}{l}\text { Minas } \\
\text { Gerais }\end{array}$ & $\begin{array}{l}\text { Apreender as possibilidades } \\
\text { de atuação junto desta } \\
\text { população diante dos } \\
\text { desafios colocados para a } \\
\text { garantia do seu acesso às } \\
\text { ações de cuidado à saúde, } \\
\text { possibilitando sistematizar } \\
\text { um panorama das ações } \\
\text { desenvolvidas, bem como } \\
\text { das lacunas assistenciais } \\
\text { existentes }\end{array}$ & $\begin{array}{c}\text { Revisão } \\
\text { integrativa/Qualit } \\
\text { ativa }\end{array}$ & $\begin{array}{l}\text { Amostra de vinte e } \\
\text { cinco trabalhos } \\
\text { acadêmicos }\end{array}$ \\
\hline E5 & $\begin{array}{l}\text { Bittencourt } \\
\text { et al. }\end{array}$ & 2019 & $\begin{array}{c}\text { Escola } \\
\text { Anna Nery }\end{array}$ & Amapá & $\begin{array}{l}\text { Analisar práticas } \\
\text { assistenciais de profissionais } \\
\text { de uma eCR sobre o cuidado } \\
\text { prestado aos usuários de } \\
\text { álcool e outras drogas em } \\
\text { Macapá }\end{array}$ & $\begin{array}{c}\text { Estudo } \\
\text { descritivo/Qualitat } \\
\text { iva }\end{array}$ & $\begin{array}{l}\text { Dez profissionais de } \\
\text { uma eCR de Macapá }\end{array}$ \\
\hline E6 & Pinto et al. & 2018 & $\begin{array}{l}\text { Revista de } \\
\text { Enfermage } \\
\text { m UFPE } \\
\text { online }\end{array}$ & $\begin{array}{c}\text { Rio } \\
\text { Grande } \\
\text { do Sul }\end{array}$ & $\begin{array}{l}\text { Apresentar as experiências } \\
\text { vividas por uma enfermeira } \\
\text { no exercício de suas funções } \\
\text { numa eCR }\end{array}$ & $\begin{array}{c}\text { Relato de } \\
\text { experiência/Qualit } \\
\text { ativa }\end{array}$ & $\begin{array}{l}\text { Enfermeira de uma } \\
\text { eCR }\end{array}$ \\
\hline
\end{tabular}




\begin{tabular}{|c|c|c|c|c|c|c|c|}
\hline E7 & Magalhães & 2018 & $\begin{array}{l}\text { Revista de } \\
\text { Psicologia } \\
\text { da UNESP }\end{array}$ & $\begin{array}{l}\text { São } \\
\text { Paulo }\end{array}$ & $\begin{array}{l}\text { Discorrer sobre os desafios } \\
\text { da construção da clínica } \\
\text { nômade, na rotina do } \\
\text { processo de trabalho das } \\
\text { equipes e no enfrentamento } \\
\text { das contradições entre a } \\
\text { prática e o proposto pela } \\
\text { portaria }\end{array}$ & $\begin{array}{c}\text { Cartografia/Qualit } \\
\text { ativa }\end{array}$ & $\begin{array}{c}\text { Duas eCR do } \\
\text { município de São } \\
\text { Paulo }\end{array}$ \\
\hline E8 & Paula et al. & 2018 & $\begin{array}{c}\text { Revista } \\
\text { Brasileira } \\
\text { de } \\
\text { Enfermage } \\
\text { m }\end{array}$ & $\begin{array}{l}\text { Rio de } \\
\text { Janeiro }\end{array}$ & $\begin{array}{l}\text { Relatar a experiência sobre a } \\
\text { implantação de um } \\
\text { equipamento clínico de } \\
\text { produção de cuidado em } \\
\text { saúde à População em } \\
\text { Situação de Rua, } \\
\text { denominado Consultório na } \\
\text { Rua }\end{array}$ & $\begin{array}{c}\text { Relato de } \\
\text { experiência/Qualit } \\
\text { ativa }\end{array}$ & $\begin{array}{c}\text { Uma eCR da zona } \\
\text { oeste do Rio de } \\
\text { Janeiro }\end{array}$ \\
\hline E9 & $\begin{array}{l}\text { Cardoso et } \\
\text { al. }\end{array}$ & 2018 & $\begin{array}{l}\text { Revista } \\
\text { Latino- } \\
\text { Americana } \\
\quad \text { de } \\
\text { Enfermage } \\
\quad \mathrm{m}\end{array}$ & Alagoas & $\begin{array}{l}\text { Analisar elementos do } \\
\text { processo de trabalho de } \\
\text { enfermagem no Consultório } \\
\text { na Rua, evidenciando os } \\
\text { desafios e potencialidades } \\
\text { do cuidado à pessoa em } \\
\text { situação de rua }\end{array}$ & $\begin{array}{l}\text { Estudo } \\
\text { exploratório/Quali } \\
\text { tativa }\end{array}$ & $\begin{array}{l}\text { Cinco enfermeiros do } \\
\text { CnaR de Maceió }\end{array}$ \\
\hline E10 & Lima e Seidl & 2017 & $\begin{array}{l}\text { Psicologia } \\
\text { em } \\
\text { Pesquisa }\end{array}$ & Goiás & $\begin{array}{l}\text { Descrever ações de Redução } \\
\text { de Danos desenvolvidas no } \\
\text { CnaR do município de } \\
\text { Goiânia, segundo relatos de } \\
\text { profissionais e de pessoas } \\
\text { atendidas }\end{array}$ & $\begin{array}{c}\text { Estudo } \\
\text { exploratório/Quali } \\
\text { tativa }\end{array}$ & $\begin{array}{c}\text { Nove profissionais de } \\
\text { uma eCR de Goiânia } \\
\text { e quatro usuários }\end{array}$ \\
\hline E11 & $\begin{array}{l}\text { Engstrom e } \\
\text { Teixeira }\end{array}$ & 2016 & $\begin{array}{l}\text { Ciência \& } \\
\text { Saúde } \\
\text { Coletiva }\end{array}$ & $\begin{array}{l}\text { Rio de } \\
\text { Janeiro }\end{array}$ & $\begin{array}{l}\text { Descrever e refletir acerca } \\
\text { das práticas de cuidado e } \\
\text { promoção da saúde de uma } \\
\text { eCR que atuava em um } \\
\text { território vulnerável em um } \\
\text { grande centro urbano }\end{array}$ & $\begin{array}{c}\text { Estudo } \\
\text { exploratório/Quali } \\
\text { tativa }\end{array}$ & $\begin{array}{l}\text { Uma eCR de } \\
\text { Manguinhos }\end{array}$ \\
\hline E12 & $\begin{array}{c}\text { Silva, Cruz e } \\
\text { Vargas }\end{array}$ & 2015 & $\begin{array}{l}\text { Saúde } \\
\text { Debate }\end{array}$ & $\begin{array}{l}\text { Rio de } \\
\text { Janeiro }\end{array}$ & $\begin{array}{l}\text { Compreender as práticas de } \\
\text { cuidado de um CnaR, em } \\
\text { Manguinhos/RJ, de forma a } \\
\text { contribuir com o debate da } \\
\text { APS para populações } \\
\text { específicas }\end{array}$ & $\begin{array}{c}\text { Estudo de } \\
\text { caso/Qualitativa }\end{array}$ & $\begin{array}{l}\text { Uma eCR de } \\
\text { Manguinhos }\end{array}$ \\
\hline E13 & Lima e Seidl & 2015 & $\begin{array}{l}\text { Psicologia } \\
\text { em Estudo }\end{array}$ & Goiás & $\begin{array}{l}\text { Investigar os modos de } \\
\text { atuação e as características } \\
\text { do trabalho de intervenção } \\
\text { com adultos jovens em } \\
\text { situação de rua e usuários de } \\
\text { substâncias psicoativas, } \\
\text { segundo percepções de } \\
\text { profissionais do CnaR do } \\
\text { município de Goiânia e de } \\
\text { pessoas usuárias atendidas } \\
\text { pelo CnaR }\end{array}$ & $\begin{array}{c}\text { Estudo } \\
\text { exploratório/Quali } \\
\text { tativa }\end{array}$ & $\begin{array}{c}\text { Nove profissionais de } \\
\text { uma eCR de Goiânia } \\
\text { e quatro usuários }\end{array}$ \\
\hline
\end{tabular}

Fonte: Dados da pesquisa (2021). 
A partir da leitura e análise dos estudos, foi possível mapear na literatura a atuação dos profissionais do CnaR. Para melhor compreensão o Quadro 2 foi elaborado e nele os resultados mapeados foram organizados em eixos, a saber: assistência à saúde; articulação com os dispositivos das Redes de Atenção à Saúde (RAS) e Redes Intersetoriais; tecnologias leves de cuidado; redução de danos; e trabalho em equipe.

Quadro 2. Resultados mapeados da atuação dos profissionais do CnaR organizados em eixos com distribuição nos estudos. Maceió, Alagoas, 2021.

\begin{tabular}{|c|c|c|}
\hline Eixos de atuação & Estudos & Principais resultados \\
\hline Atenção à saúde & $\begin{array}{c}\text { E1, E2, E3, E4, } \\
\text { E6, E7, E8, E9, } \\
\text { E11, E12, E13 }\end{array}$ & $\begin{array}{l}\text { Atividades desenvolvidas in loco (na rua) com entrevistas e investigações no território que } \\
\text { possibilitaram identificar a condição de vulnerabilidade e situação de rua (E1, E4, E7, E8, E9, } \\
\text { E10, E13); } \\
\text { Visitas sistemáticas ao território (E1, E3, E7, E9, E10, E11, E12); } \\
\text { Atividades de promoção, prevenção e manejo dos agravos clínicos (E1, E3, E6, E11); } \\
\text { Encaminhamento para consultas, exames e procedimentos (E2; E3; E5; E7, E8, E9, E11); } \\
\text { Dispensação de medicamentos (E3, E4, E5, E7, E8, E11); } \\
\text { Primeiros cuidados nas urgências e emergências (E3, E4, E7); } \\
\text { Atendimentos de psicologia (E3, E5, E8, E11); } \\
\text { Atendimentos com Assistente Social (E3, E4, E8, E11); } \\
\text { Consultas de enfermagem (E3, E6, E8); } \\
\text { Consultas médica (E3, E5, E8); } \\
\text { Acolhimento da demanda espontânea e programada (E1; E4; E7, E9, E12, E13); } \\
\text { Acompanhamento e tratamento da tuberculose (E2, E3, E8, E11); } \\
\text { Acompanhamento e tratamento de doenças crônicas não-transmissíveis (hipertensão e diabetes) } \\
\text { (E3); } \\
\text { Acompanhamento de usuário de saúde mental (E4, E7, E11); } \\
\text { Ações no campo da saúde bucal com avaliações, orientações e tratamento clínicos (E3, E4, E11); } \\
\text { Atendimento de pré-natal (E2, E3, E8, E9); } \\
\text { Acompanhamento no puerpério (E3); } \\
\text { Testes de gravidez (E3, E11); } \\
\text { Coleta de exame citopatológico (E11); } \\
\text { Cuidado às pessoas com IST, HIV/AIDS (E3, E8); } \\
\text { Oferta de testes rápidos para sífilis, HIV e Hepatites B e C (E6, E8, E11); } \\
\text { Tratamento para sífilis (E3); } \\
\text { Realização de curativos (E2, E3, E4, E8, E11); } \\
\text { Mensuração de glicemia capilar (E8, E9); } \\
\text { Aferição de pressão arterial (E8, E9); } \\
\text { Vacinação (E11). }\end{array}$ \\
\hline $\begin{array}{l}\text { Articulação com } \\
\text { dispositivos das } \\
\text { Redes de Atenção à } \\
\text { Saúde (RAS) e } \\
\text { Redes Intersetoriais }\end{array}$ & $\begin{array}{l}\text { E1, E3, E4, E5, } \\
\text { E6, E7, E8, E11 }\end{array}$ & $\begin{array}{l}\text { RAS: } \\
\text { Centros de atenção psicossocial (CAPS), incluindo o específico para Álcool e outras Drogas } \\
\text { (CAPS-AD) (E1, E4, E5, E6, E7, E8, E11); } \\
\text { Hospitais (E1, E3, E4, E5, E8, E9, E11); } \\
\text { Unidade Básicas de Saúde (UBS) (E4, E5, E6, E7, E11); } \\
\text { Serviço Móvel de Urgência e Emergência (SAMU) (E1, E6, E11); } \\
\text { Unidades de Pronto Atendimento (UPA) (E6, E11); } \\
\text { Unidade Básica de Atendimento Imediato (UBAI) (E6); } \\
\text { Policlínica (E4). }\end{array}$ \\
\hline
\end{tabular}




\begin{tabular}{|c|c|c|}
\hline & & $\begin{array}{l}\text { Redes Intersetoriais: } \\
\text { Centro de Referência Especializado para Pessoas em Situação de Rua (Centro POP) (E1, E5, } \\
\text { E6); } \\
\text { Centro de Referência Especializada de Assistência Social (CREAS) (E7, E8, E11); } \\
\text { Abrigos/Albergues (E3, E7, E11); } \\
\text { Secretarias de trabalho e habitação (E7); } \\
\text { Centros de convivência (E7); } \\
\text { Promotoria municipal (E1); } \\
\text { Cartórios (E1); } \\
\text { Instituto Nacional do Seguro Social (INSS) (E1); } \\
\text { Bolsa Família (E3); } \\
\text { Benefícios de transporte urbano (E1, E3) } \\
\text { Benefício de prestação continuada (E3) } \\
\text { Articulação para retirada de documentos (E4, E5, E11). }\end{array}$ \\
\hline $\begin{array}{c}\text { Tecnologias leves } \\
\text { de cuidado }\end{array}$ & $\begin{array}{c}\text { E1, E2, E3, E4, } \\
\text { E5, E8, E9, } \\
\text { E10, E12 e E13 }\end{array}$ & $\begin{array}{l}\text { Construção de vínculo (E1, E3, E4, E5, E7, E8, E9, E10, E11, E12, E13); } \\
\text { Escuta qualificada (E3; E4, E6, E8, E9, E10, E11, E12, E13); } \\
\text { Acolhimento (E1, E3, E7, E8, E9, E11, E12, E13); } \\
\text { Empatia (E1, E9); } \\
\text { Desenvolvimento de atividades utilizando música (arte) e futebol (lazer) (E2, E13). }\end{array}$ \\
\hline Redução de danos & $\begin{array}{c}\text { E1, E2, E3, E5, } \\
\text { E9, E10, E11, } \\
\text { E12 e E13 }\end{array}$ & $\begin{array}{l}\text { Não exigência da interrupção do uso de Substancias Psicoativas (SPA) (R10); } \\
\text { Posição respeitosa (E2, E3, E9, E10); } \\
\text { Redução do preconceito (E10, E11, E13); } \\
\text { Minimização de sofrimento (E10); } \\
\text { Consideração da subjetividade e valorização do outro (E1, E4, E9, E10, E12); } \\
\text { Promoção da autonomia do sujeito (E1, E3, E7, E9, E11); } \\
\text { Fortalecimento da autoestima (E1, E9, E11); } \\
\text { Empoderamento do sujeito (E7); } \\
\text { Valorização da cidadania (E1, E3, E11); } \\
\text { Distribuição de água e insumos como preservativos (E1, E2, E4, E11); } \\
\text { Distribuição de material gráfico com informações sobre Infecções Sexualmente Transmissíveis } \\
\text { (IST) e uso de SPA (E1; E13). }\end{array}$ \\
\hline Trabalho em equipe & $\begin{array}{c}\text { E1, E3, E7, E9, } \\
\text { E11, E12 }\end{array}$ & $\begin{array}{l}\text { Atuação em equipe multiprofissional e interdisciplinar (E1, E3, E7, E9, E12); } \\
\text { Reunião de equipe para discussão de casos e temas (E3, E7, E12); } \\
\text { Construção de Projeto Terapêutico Singular (PTS) (E3, E11); } \\
\text { Processos constantes de troca de saberes e técnicas entre os profissionais da equipe (E12); } \\
\text { Elaboração de Planejamento Estratégico em Saúde (E9); } \\
\text { Planejamento das ações (E11); } \\
\text { Reunião conjunta com CREAS (E11). }\end{array}$ \\
\hline
\end{tabular}

Fonte: Dados da pesquisa (2021).

No que tange ao eixo de atuação ‘Atenção à saúde', compreendem-se as ações, serviços e cuidados prestados. Mapeouse que as atividades promovidas pelos profissionais são desenvolvidas in loco (na rua) com entrevistas e investigações no território que possibilitam identificar a condição de vulnerabilidade e situação de rua (E1, E4, E7, E8, E9, E10, E13). Estas se dão a partir de visitas sistemáticas aos territórios (E1, E3, E7, E9, E10, E11, E12) com atividades de promoção, prevenção e 
manejo dos agravos clínicos (E1, E3, E6, E11). Por vezes, para a garantia do cuidado integral, faz-se necessário o encaminhamento dos usuários para consultas, exames e procedimentos (E2; E3; E5; E7, E8, E9, E11).

Ademais, encontram-se ainda nesse eixo o acolhimento da demanda espontânea e programada (E1; E4; E7, E9, E12, E13). Ocasionalmente, os profissionais necessitam prestar os primeiros cuidados nas urgências e emergências junto a essa população (E3, E4, E7). Dentro do rol de serviços, podem-se ser verificados os atendimentos de psicologia (E3, E5, E8, E11) e os realizados com Assistente Social (E3, E4, E8, E11), bem como as consultas de enfermagem (E3, E6, E8) e médicas (E3, E5, E8). Também se observaram ações no campo da saúde bucal com avaliações, orientações e tratamento clínicos (E3, E4, E11). No que se refere ao acompanhamento e tratamento de doenças infectocontagiosas e doenças crônicas transmissíveis ou não, foram verificadas a tuberculose (E2, E3, E8, E11), hipertensão e diabetes (E3).

Acrescentado a estas ações e serviços, destacaram-se o acompanhamento de usuário de saúde mental (E4, E7, E11) e os atendimentos de pré-natal (E2, E3, E8, E9) e puerperal (E3). Quando mencionado o cuidado às pessoas com Infecções Sexualmente Transmissíveis (IST) incluindo HIV e a Síndrome da Imunodeficiência Adquirida (AIDS) (E3, E8), observa-se que um leque de outros serviços é prestado. Dentre estes, apresentam-se a oferta de testes rápidos para sífilis, HIV e Hepatites B e C (E6, E8, E11) e o tratamento para sífilis (E3). No tocante à atuação mais específica da enfermagem, assinalam-se a realização de curativos (E2, E3, E4, E8, E11), mensuração de glicemia capilar (E8, E9), aferição de pressão arterial (E8, E9), vacinação (E11), coleta de exame citopatológico (E11) e a realização de testes de gravidez (E3, E11).

No que concerne ao eixo 'Articulação com dispositivos das RAS e Redes Intersetoriais', verificam-se que os Centros de Atenção Psicossocial (CAPS), incluindo os específicos para Álcool e outras Drogas (CAPS-AD) (E1, E4, E5, E6, E7, E8, E11) são os registrados com maior frequência. Seguindo de articulação com outros pontos de serviços da atenção secundária e terciária: os hospitais (E1, E3, E4, E5, E8, E9, E11), Serviço Móvel de Urgência e Emergência (SAMU) (E1, E6, E11), Unidades de Pronto Atendimento (UPA) (E6, E11), e Policlínica (E4). Dentre os serviços da APS, destacaram-se ainda as Unidades Básicas de Saúde (E4, E5, E6, E7, E11) e a Unidade Básica de Atendimento Imediato (UBAI) (E6).

Do mesmo modo, os profissionais do CnaR articulam com outros setores para além da saúde com destaque para o Centro de Referência Especializado para Pessoas em Situação de Rua (Centro POP) (E1, E5, E6) e o Centro de Referência Especializado de Assistência Social (CREAS) (E7, E8, E11). Os abrigos e albergues também são serviços com que os profissionais atuam (E3, E7, E11). O trabalho desenvolvido pelos Assistentes Sociais do CnaR funciona na perspectiva da garantia de direitos. Em meio a estes, assinalam-se a articulação para retirada de documentos (E4, E5, E11), Bolsa Família (E3), benefícios de transporte urbano (E1, E3) e o Benefício de Prestação Continuada (BPC) (E3). Nesse contexto, também são verificadas articulações com as secretarias de trabalho e habitação (E7), centros de convivência (E7), Promotoria municipal (E1), cartórios (E1) e o Instituto Nacional do Seguro Social (INSS) (E1).

Quanto ao eixo intitulado 'Tecnologias leves de cuidado', encontram-se evidentes a construção de vínculo (E1, E3, E4, E5, E7, E8, E9, E10, E11, E12, E13), escuta qualificada (E3; E4, E6, E8, E9, E10, E11, E12, E13), e o acolhimento (E1, E3, E7, E8, E9, E11, E12, E13). Além destas, foram assinaladas a empatia (E1, E9) e o desenvolvimento de atividades utilizando música (arte) e futebol (lazer) (E2, E13).

A prática da Redução de Danos (RD) está totalmente ligada ao uso das tecnologias leves de cuidado. Uma frequente característica relacionada à PSR é o uso e abuso de SPAs. Nesse ínterim emerge o eixo 'Redução de danos', que vai discorrer sobre a não exigência da interrupção do uso de SPAs (R10) para a prestação do cuidado. Foram verificadas algumas ações no campo da RD, dentre elas podem ser citadas, a consideração da subjetividade e valorização do outro (E1, E4, E9, E10, E12), promoção da autonomia do sujeito (E1, E3, E7, E9, E11), fortalecimento da autoestima (E1, E9, E11), valorização da cidadania (E1, E3, E11) e a promoção do empoderamento do sujeito (E7). Para tanto, os profissionais assumem uma posição respeitosa (E2, E3, E9, E10) que caminha para a redução do preconceito (E10, E11, E13) e minimização de sofrimento (E10) de quem faz 
o uso. Outras ações mapeadas foram a distribuição de água e insumos como preservativos (E1, E2, E4, E11), bem como distribuição de material gráfico com informações sobre Infecções Sexualmente Transmissíveis (IST) e uso de SPAs (E1; E13).

$\mathrm{O}$ quarto eixo compreende ações que acontecem em equipe. Dentre estas, destacaram-se a atuação em equipe multiprofissional e interdisciplinar (E1, E3, E7, E9, E12) e as reuniões de equipe para discussão de casos e temas (E3, E7, E12). Um dos estudos mapeados revelou a experiência de reuniões conjuntas com o CREAS (E11). Observou-se ainda que existem processos constantes de troca de saberes e técnicas entre os profissionais da equipe (E12) com construção de Projeto Terapêutico Singular (PTS) (E3, E11). Ademais, foram verificadas atuações para planejamento das ações (E11) e elaboração de Planejamento Estratégico em Saúde (PES) no contexto do CnaR (E9).

\section{Discussão}

Os primeiros dados que subsidiam esta discussão são o baixo quantitativo de estudos publicados com concentração nos anos de 2018 e 2019 e a centralidade da temática no país nas regiões Sudeste e Centro-Oeste. Estes fatos revelam que o assunto tem sido abordado mais recentemente. Cardoso et al. (2018) apontaram em sua pesquisa a escassez de estudos relativos à atuação dos profissionais do CnaR. Tais circunstâncias podem estar relacionadas aos processos de autorização e implementação das eCR acontecerem muito lentamente, embora existam algumas estratégias de apoio para sua efetivação por parte do Ministério da Saúde (MS) (Medeiros \& Cavalcante, 2018). Acrescente-se ainda que, o processo de ampliação das eCR não aconteceu no Brasil de forma homogênea e teve seu início nos grandes centros urbanos, uma vez que a normativa contemplava apenas municípios acima de cem mil habitantes e que possuíam um mínimo de oitenta pessoas em situação de rua (Brasil, 2012).

Mapear a literatura permitiu encontrar os resultados dessa atuação e estes foram categorizados em eixos. Diante dos dados apresentados no eixo "Assistência à Saúde", os estudos demonstraram que as ações e serviços prestados acontecem no âmbito da rua. Esta dinâmica de funcionamento itinerante in loco é corroborada por Duarte (2019) e Medeiros e Cavalcante (2018) em suas pesquisas. Ressalta-se a respeito da importância do desenvolvimento das atividades nos territórios onde a população se encontra, uma vez que estes são seus locais de produção de vida (Silva Junior \& Belloc, 2018). De acordo com Engstrom e Teixeira (2016), as visitas sistemáticas aos territórios dizem respeito à abordagem territorializada em que se busca não apenas reconhecer as características geográficas, mas também as sanitárias, ambientais, áreas de risco e violência.

Os estudos que compuseram o primeiro eixo também evidenciaram um vasto leque de serviços assistenciais ofertados à PSR. Estes já estão previstos no contexto da APS e estão descritos em diversas normativas, portarias e políticas, a exemplo a PNAB (Brasil, 2017). Entretanto, põe-se em discussão um dos grandes problemas enfrentados por essa população: o acesso aos serviços de saúde. Uma revisão de literatura elucidou os motivos que impedem que os usuários procurem um serviço de saúde, dentre eles destacam-se "o preconceito e a discriminação relacionados às condições de higiene, que está prejudicada através do uso de vestimentas sujas, falta de banho, odor fétido e o grande tempo de espera pelo atendimento" (Silva, Silva \& Andrade, 2019).

Opondo-se às experiências negativas relacionadas às barreiras encontradas, a pesquisa de Cervieri et al. (2019) revelou que os atendimentos em saúde a essa população não são negados. Tal fato representa uma característica local em que os usuários referiram ter os documentos e/ou cartão do SUS, sendo este um fator importante para o acesso (Cervieri et al., 2019). Inserido nesse contexto de não garantia total do acesso, Valle e Farah (2020) mencionam que o CnaR possui papel primordial nesta garantia de direito à saúde que é pressuposto essencial para a dignidade desse grupo. Então, infere-se que os profissionais do CnaR possuem uma atuação que consolida no cotidiano da PSR o princípio da Universalidade do SUS.

Para que se forneça a essa população um cuidado integral e equânime, faz-se necessário a articulação do CnaR com outros dispositivos das RAS e Redes Intersetoriais. No que concerne a esse eixo de atuação, o conjunto de resultados apresentados 
trazem uma relação de serviços em sua maioria relacionados ao Serviço Social. "As ações intersetoriais possibilitam o atendimento integral e visam à superação das fragmentações decorrentes das estruturas setorializadas, viabilizando a atenção às necessidades sociais da população" (Veridiano, Andrade \& Gomes, 2017). Frente aos resultados encontrados, evidenciou-se uma maior articulação do CnaR com o Centro POP e CREAS, serviços ligados à Rede de Proteção Social Especial de média complexidade. Pinto et al. (2018) relatam a experiência de uma profissional do CnaR em articulação com o Centro POP evidenciando a possibilidade de atuação de maneira interdisciplinar para melhor assistir as necessidades do sujeito.

Com a finalidade de garantir resolutividade às demandas da PSR, faz-se necessário uma forte articulação de múltiplas ações entre serviços e setores que estão para além da saúde. "Contudo, esse imperativo que emerge do processo de trabalho, muitas vezes perde-se nas desarticulações" (Magalhães, 2018). Quando se faz menção ao atributo coordenação do cuidado pela eCR, Engstrom e Teixeira (2016) o verificaram como sendo de difícil execução, visto que dependia de redes, tanto do setor saúde, como intersetoriais. Magalhães (2018) afirma que as fragilidades destas potencializam as dificuldades enfrentadas pela PSR. Ressalta-se ainda que, dentre os serviços da RAS, o CnaR também compartilha os cuidados com os CAPS, incluindo o AD. O Ministério da Saúde aponta para um dado em que 35,5\% da PSR do país referem o uso abusivo de álcool e outras drogas como principal motivação para estarem em situação de rua. Tal fato pode estar relacionado a frequente necessidade de articulação do CnaR com o CAPS-AD (Brasil, 2014).

Em virtude da multiplicidade e das singularidades que envolvem o cuidado junto à PSR se faz necessário o entendimento sobre as tecnologias de cuidado. Dos estudos mapeados nesta revisão, depreendeu-se um eixo intitulado "Tecnologias leves de cuidado". Segundo Merhy et al. (2019), o trabalho em saúde é tecnológico sendo construído e efetivado mediante ao uso das tecnologias leves, leve-duras e duras. Com o intuito de assegurar o cuidado integral à PSR, os profissionais do CnaR precisam colocar em prática tais tecnologias o que, consequentemente, torna complexo e singular os seus processos de trabalho (Timóteo et al., 2020). Merhy et al. (2019) colocam que tudo que for utilizado para favorecer e potencializar o encontro com o outro pode ser denominado tecnologia leve, destacando-se a escuta, empatia, reconhecimento e porosidade. Os estudos demonstraram que tais tecnologias fazem parte do eixo de atuação dos profissionais. Destaca-se também o desenvolvimento de atividades que utilizam a música. Todo esse aparato tecnológico instrumentaliza o processo de trabalho das eCR que torna o cuidado prestado complexo e dinâmico.

Um estudo recente sobre a caracterização do processo de trabalho e das ações desenvolvidas pelas eCR de Maceió revelou que a primeira e principal característica identificada foi o desenvolvimento de ações norteadas pela Política de RD (Timóteo et al., 2020). Partindo desse pressuposto e observando os estudos, surgiu o eixo de atuação intitulado "Redução de danos". Uma grande parte das intervenções de RD percorrem os caminhos propostos nas tecnologias leves em que o acolhimento e vínculo são fundamentais para a construção compartilhada de projetos existenciais. De acordo com a Associação Internacional de Redução de Danos (Ihra, 2021), a RD é um conjunto de políticas e práticas cujo objetivo é mitigar danos biopsicossociais associados ao uso de SPAs em pessoas que não querem, não podem ou não conseguem parar o uso. Como citado anteriormente, as questões relacionadas às SPAs têm ligação com a PSR.

As intervenções de âmbito da RD acontecem de modo a considerar a subjetividade e a individualidade do sujeito, bem como promover o autocuidado e a autonomia. Frente aos resultados encontrados na literatura, foi possível identificar outras ações importantes para o cuidado junto às pessoas que fazem uso de substâncias, dentre elas podem ser destacadas a distribuição de água e insumos como preservativos (Souza et al., 2020) e a material gráfico com informações sobre IST e uso de SPAs (Lima \& Seidl, 2015). Entretanto, existe uma realidade na atuação desses profissionais que diz respeito à escassez de insumos como verificados nos estudos de Timóteo et al. (2020) e Lima e Seidl (2015).

O quarto e último eixo a ser discutido nesta revisão de escopo é o "Trabalho em equipe". As eCR são compostas por profissionais de diferentes áreas que trabalham numa perspectiva multiprofissional (Timóteo et al., 2020). A portaria 1.029, de 
20 de maio de 2014 é a que estabelece o rol de categorias profissionais que podem compor as equipes e distingue as três modalidades (Brasil, 2014). Cardoso et al. (2018) colocam que para contornar as dificuldades e adversidades encontradas no cuidado às PSR, o trabalho em saúde deve ser organizado em uma perspectiva multiprofissional e interdisciplinar. Mapeou-se também atuação relacionada às reuniões de equipe para planejamento de ações bem como para discussão de casos e elaboração de PTS.

\section{Conclusão}

O total de publicações incluídas nesta revisão de escopo demonstra que a temática em estudo tem sido pouco discutida. Nesse contexto, diante dos achados suscita-se a demanda por pesquisas que tenham como finalidade elucidar a atuação dos profissionais do CnaR. Os resultados do estudo promovem inovação, divulgação e disseminação dessa temática que é de grande relevância para a saúde pública no Brasil, uma vez que elenca, mapeia e categoriza em eixos a atuação dos referidos profissionais. Por fim, chama-se a atenção para que as futuras pesquisas sejam realizadas com maior rigor metodológico, a fim de configurar um cenário de produção científica qualificada.

\section{Referências}

Barbiani, R., Nora, C., \& Schaefer, R. (2016). Práticas do enfermeiro no contexto da atenção básica: scoping review. Revista Latino-Americana de Enfermagem. 24(1), 1-12.

Bittencourt, M. N. et al. (2019). Street clinic: the care practices with users of alcohol and other drugs in Macapá. Escola Anna Nery, 23(1), 1-7.

Brasil (2011). Portaria $n^{\circ}$ 2488, de 21 de outubro de 2011. Aprova a Política Nacional de Atenção Básica, estabelecendo a revisão de diretrizes e normas para a organização da Atenção Básica, para a Estratégia Saúde da Família (ESF) e o Programa de Agentes Comunitários de Saúde (PACS).

Brasil (2014). Saúde da população em situação de rua: um direito humano. Ministério da Saúde.

Brasil (2013). Portaria $n^{\circ} 2436$, de 21 de setembro de 2017. Aprova a Política Nacional de Atenção Básica, estabelecendo a revisão de diretrizes para a organização da Atenção Básica, no âmbito do Sistema Único de Saúde (SUS).

Brasil (2012). Portaria $n^{\circ}$ 123, de 25 de janeiro de 2012. Define os critérios de cálculo do número máximo de equipes de Consultório na Rua (eCR) por Município.

Brasil (2014). Portaria $n^{\circ}$ 1029, de 20 de maio de 2014. Amplia o rol das categorias profissionais que podem compor as Equipes de Consultório na Rua em suas diferentes modalidades e dá outras providências.

Cardoso, A. C., et al. (2018). Challenges and potentialities of nursing work in street medical office. Revista Latino-Americana de Enfermagem, 26, 1-9.

Cervieri, N. B., et al. (2019). O acesso aos serviços de saúde na perspectiva de pessoas em situação de rua. Smad Revista Eletrônica Saúde Mental Álcool e Drogas, 15 (4), 1-8.

Duarte, A. H. C. (2019). O Acolhimento em saúde no espaço da rua: estratégias de cuidado do Consultório na Rua. Textos \& Contextos (Porto Alegre), 18(2) p.34306.

Engstrom, E. M., et al. (2019). A dimensão do cuidado pelas equipes de Consultório na Rua: desafios da clínica em defesa da vida. Saúde Em Debate, 43(spe7), $50-61$.

Engstrom, E. M., \& Teixeira, M. B. (2016). Equipe "Consultório na Rua” de Manguinhos, Rio de Janeiro, Brasil: Práticas de cuidado e promoção da saúde em um território vulnerável. Ciencia e Saude Coletiva, 21(6), 1839-1848.

Hallais, J. A. da S., \& de Barros, N. F. (2015). Consultório na Rua: Visibilidades, invisibilidades e hipervisibilidade. Cadernos de Saúde Pública, 31(7), 14971504.

Hria (2021). Harm Reduction International Association (United Kingdom). Harm reduction. 2021. Disponível em: https://www.hri.global/about. Acesso em: 10 fev de 2021.

Jbi, Joanna Briggs Institute (2020). The Joanna Briggs institute reviewers' manual 2020: methodology for JBI scoping reviews. South Australia: The Joanna Briggs Institute.

Lima, H. S., \& Seidl, E. M. F. (2017). Consultório na Rua: percepção de profissionais e de usuários sobre redução de danos. Revista Psicologia Em Pesquisa, 11(2), 33-41.

Lima, H. S., \& Seidl, E. M. F. (2015). Street outreach office: Attention to people on psychoactive drug/substance abuse. Psicologia em Estudo, $20(1), 57-69$. 
Research, Society and Development, v. 10, n. 4, e59410414470, 2021

(CC BY 4.0) | ISSN 2525-3409 | DOI: http://dx.doi.org/10.33448/rsd-v10i4.14470

Magalhães, J. A. (2018). Clínica do consultório na rua: Atenção à população em situação de rua no centro da cidade de. Revista de Psicologia da UNESP, 17(1), $14-33$.

Medeiros, C. R. S., \& Cavalcante, P. (2018). The implementation of the Brazilian health program for the homeless population - Consultório na Rua: Obstacles and advantages. Saúde e Sociedade, 27(3), 754-768.

Merhy, E. E., et al. (2019). Rede Básica, campo de forças e micropolítica: implicações para a gestão e cuidado em saúde. Saúde em Debate, 43(6), p. 70-83.

Paula, H. C. et al. (2018). A implantação do Consultório na Rua na perspectiva do cuidado em saúde. Revista Brasileira de Enfermagem, 71(6), $2843-2847$.

Pinto, A. H., et al. (2018). Determinantes sociais, equidade e consultório na rua. Revista de Enfermagem UFPE on Line, 12(12), 3513.

Vale, A. R., \& Vecchia, M. D. (2019). The health care of people living in the streets: Possibilities and challenges. Estudos de Psicologia, 24(1), 42-51.

Valle, F. A. A. L., \& Farah, B. F. (2020). A saúde de quem está em situação de rua: (in)visibilidades no acesso ao sistema único de saúde. Physis: Revista de Saúde Coletiva, (30) 2, p. 1-21.

Veridiano, A. L., Andrade, L. de, \& Gomes, A. H. (2017). Práticas intersetoriais na atenção às pessoas em situação de rua: uma atuação entre "saúde" e "assistência social." Visão, 6(2), 115-166.

Santos, C. F., \& Ceccim, R. B. (2018). Encontros na rua: possibilidades de saúde em um consultório a céu aberto. Interface - Comunicação, Saúde, Educação, 22(67), 1043-1052.

Silva, C. C. da, Cruz, M. M. da, \& Vargas, E. P. (2015). Práticas de cuidado e população em situação de rua: o caso do Consultório na Rua. Saúde Em Debate, $39,246-256$.

Silva, B. M., Silva, V. N., \& Andrade, E.G.S. (2019). Dificuldades encontradas pelos moradores de rua no acesso à saúde pública. Rev Inic Cient Ext. 2019; 2(2), $280-286$.

Silva Junior, D.V. \& Belloc, M. M. (2018). Habitar invisível: produção de vida e cuidado na experiência urbana. Interface- Comunicação, Saúde, Educação, 22(67), 1065-1075.

Souza, A. C. C. et al. (2020). Redução de vulnerabilidades como estratégia de cuidados do consultório na rua. Revista do NUFEN, (12)3, 103-115.

Teixeira, M. B. et al. (2019). Os invisibilizados da cidade: o estigma da população em situação de rua no rio de janeiro. Saúde em Debate, (43) 7, 92-101.

Timóteo, A. V. G., et al. (2020). Caracterização do trabalho e ações desenvolvidas pelas equipes do Consultório na Rua de Maceió - AL. Enfermagem Em Foco, 11(1), 126-130. 